\title{
Morphology control of the magnetization reversal mechanism in $\mathrm{Co}_{80} \mathrm{Ni}_{20}$ nanomagnets
}

\author{
Silvana Mercone ${ }^{1}$ * Fatih $_{\text {Zighem }}{ }^{1}$, Brigitte Leridon ${ }^{2,3,4}$, \\ Audrey Gaul ${ }^{1}$, Frédéric Schoenstein ${ }^{1}$, and Noureddine Jouini ${ }^{1}$ \\ ${ }^{1}$ Laboratoire des Sciences des Procédés et des Matériaux (UPR3407), \\ CNRS-Université Paris XIII, Sorbonne Paris Cité, Villetaneuse, France \\ ${ }^{2}$ CNRS-UMR8213, Laboratoire de Physique et d'étude des Matériaux, \\ 10 Rue Vauquelin, F-75231 Paris cedex 5, France \\ ${ }^{3}$ ESPCI-ParisTech, LPEM, 10 Rue Vauquelin, F-75231 Paris cedex 5, France and \\ ${ }^{4}$ Sorbonne Université, UPMC, LPEM, 10 Rue Vauquelin, F-75231 Paris cedex 5, France
}

(Dated: July 28 2014)

\begin{abstract}
Nanowires with very different size, shape, morphology and crystal symmetry can give rise to a wide ensemble of magnetic behaviors whose optimization determines their applications in nanomagnets. We present here an experimental work on the shape and morphological dependence of the magnetization reversal mechanism in weakly interacting $\mathrm{Co}_{80} \mathrm{Ni}_{20}$ hexagonal-close-packed nanowires. Non-agglomerated nanowires (with length $L$ and diameter $d$ ) with a controlled shape going from quasi perfect cylinders to diabolos, have been studied inside their polyol solution in order to avoid any oxidation process. The coercive field $H_{C}$ was found to follow a standard behavior and to be optimized for an aspect ratio $\frac{L}{d}>15$. Interestingly, an unexpected behavior was observed as function of the head morphology leading to the strange situation where a diabolo shaped nanowire is a better nanomagnet than a cylinder. This paradoxical behavior can be ascribed to the growth-competition between the aspect ratio $\frac{L}{d}$ and the head morphology ratio $\frac{d}{D}$ ( $D$ being the head width). Our experimental results clearly show the importance of the independent parameter $(t=$ head thickness) that needs to be considered in addition to the shape aspect ratio $\left(\frac{L}{d}\right)$ in order to fully describe the nanomagnets magnetic behavior. Micromagnetic simulations well support the experimental results and bring important insights for future optimization of the nanomagnets morphology.
\end{abstract}

PACS numbers:

Keywords: Co nanowires, coercive field, magnetization curves, morphology optimization.

\section{INTRODUCTION}

Since the development of a wide range of synthesis routes for the elaboration of controlled nano-objects, the number of studies trying to understand the zoology of the magnetic properties showed by these systems is continuously increasing 1 , 9. Among them, Cobased anisotropic nanomaterials are attracting a great part of scientific interest mainly for their possible applications as nanomagnets in (nano)medicine 10 and recording media[11, and as building blocks in high energy nanostructured bulk magnets[12]. All these studies showed that depending on the elaboration process, the nanowires can present very different size, shape, morphology and crystal symmetry as well as very different as-grown spacing between the nano-objects[7, 14, 22]. Thus, altogether, those parameters give rise to a wide ensemble of magnetic behaviors that are far from being fully controlled. For Co-based nanowires, a huge issue is the fine control of their shape and morphology and how these characteristics influence the magnetic properties 1 3, 9, 20, 22, 26, 27]. Indeed, the experimental probe of the coercivity behavior as function of the shape and morphology is mandatory for a complete understanding of the magnetization reversal mechanism in these nano-objects

*Electronic address: silvana.mercone@univ-paris13.fr and thus for their applications

Experimental [3, 7, 8, 28] and theoretical[29] proofs of the influence of the crystal symmetry (hcp and fcc) of Co based nanostructures on the effective anisotropy, have been widely reported for both the magnetocrystalline term and the shape one. These terms have demonstrated a big influence on the uniformity of the magnetization reversal mechanism 3, 7, 8]. It is now also well established that length and diameter size can play an important role over the static magnetic properties of different shaped nanowires (e.g. coercivities meltdown can be easily observed with increasing diameter and decreasing length of the nanowires) [3, 22, 27. Studies of the head-morphology effect on the reversal mechanism are less evident and, to our knowledge, still an experimental open issue. This is mainly due to the difficulties of the fine control of the nanowire shape and morphology during their elaboration. Polyol process has demonstrated in the last decades, a very good control of the shape and crystallinity of nano-objects based on ferromagnetic $3 \mathrm{~d}$ metal ions(author?) [30]. This process has attracted a lot of attention thanks to its low cost together with the easy and reliable control shown over the nano-objects composition, shape and dispersion. In the case of Co based nanowires the polyol process leads to a very specific shape and morphology control that we used in this work in order to study into details the shape and morphology effect over the magnetic properties of non-agglomerated (weak interacting) $\mathrm{Co}_{80} \mathrm{Ni}_{20}$ pure hexagonal-close-packed 
nanowires.

\section{EXPERIMENTAL DETAILS}

Non-agglomerated $\mathrm{Co}_{80} \mathrm{Ni}_{20}$ nanowires have been fabricated via the polyol process route which simply consists in the reduction of metallic salts in liquid polyol(author?) [5, 13, 24. This method has shown an accurate size control of the nanoparticles via the kinetic control of the growth step (author?) [6, 24]. Briefly, in this soft chemistry route, the liquid polyol acts as a solvent and as a reducing agent for the metallic cation. By selecting a polyol with a suitable boiling point, the temperature reaction can be adjusted over a wide range of temperature letting this elaboration method to be very suitable for industrial transfer technology. In our case we used 1,2-butanediol [BEG] polyol (boiling temperature $=465 \mathrm{~K}$, dielectric constant $\left.=22.4 \mathrm{pF} \cdot \mathrm{m}^{-1}\right)$. Different morphologies have been obtained by mixing various concentration of Ruthenium chloride $(\mathrm{RuCl} 3)$ (i.e. between $0.0018 \mathrm{M}$ and $0.0048 \mathrm{M}$ ) to $0.15 \mathrm{M} \mathrm{NaOH}$ in BEG. Once Cobalt $(0.08 \mathrm{M})$ and Nickel $(0.08 \mathrm{M})$ precursors are dissolved in the mixture, this latter is then heated up to $453 \mathrm{~K}$ with a controlled temperature ramp of $279 \mathrm{~K} \cdot \mathrm{min}^{-1}$ leading to $\mathrm{Co}_{80} \mathrm{Ni}_{20}$ one-dimensional nanostructures.

Standard TEM imaging and X-Ray diffraction (XRD) patterns have been performed for all the elaborated systems. Results obtained for some of the elaborated nanowires are reported in Figure 1 (a) and (b). TEM images highlighted the necessity of four appropriate morphological parameters: i) the diameter $d$ in the middle of the nanowire, ii) the length $L$ of the nanowire, iii) the width $D$ of the head of the nanowire and iv) the thickness $t$ of the head. Accurate measurements of these four morphological parameters have been performed by measuring more than 150 pictured nanowires per batch. Histograms of each parameter (author?) 33] (not shown here) have shown typical log-normal distribution confirming for each batch a good monodispersion. Typical dispertion on $D, t$ and $d$ parameters has been found of \pm 1 $\mathrm{nm}$ while for $L$ the dispertion could be larger and depending on the batch could be found up to $\pm 10 \mathrm{~nm}$. The good control of the morphology by the polyol synthesis, allowed us to obtain very long nanowire $(L \gg d)$ as well as short ones $(L \sim 2 d)$ (see Figure 1) (a)) with morphologies varying from the cylinder type (i.e. $d=D$ ) to the diabolo one (i.e. $D>d$ ). TEM analysis gives typical morphological parameters laying in the following ranges: $L=(65-160) \mathrm{nm}, D=(8-24) \mathrm{nm}, t=(7-14) \mathrm{nm}$, $d=(7-12) \mathrm{nm}$ (author?) 33.

$\mathrm{X}$-Ray diffraction patterns have shown pure hexagonal close-packed (hcp) crystal phase for all elaborated nanowires (see Figure 1(b)) with presumably $c$-axis of the hexagonal symmetry (i.e. (001)) parallel to the nanowires length.

Using a Quantum Design MPMS 3 magnetometer, we measured standard zero field cooled (ZFC) and field cooled (FC) hysteresis cycles at different temperatures. Each ZFC magnetic loop was performed after cooling down the nanowires in the polyol solution under zero magnetic field while the FC loops were performed after cooling the same sample under the application of $H=70$ $\mathrm{kOe}$. Note that, in this latter conditions a huge percentage of nanowires is expected to align along the applied magnetic field before the polyol solution freezes. Indeed all our FC cycles have been performed with a magnetic field parallel to the length $L$ of the blocked nanowires (i.e. magnetic field parallel to the easy axis of the nanowires). The probed temperature range was between $200 \mathrm{~K}$ and 20K. The upper temperature limit was fixed by the freezing temperature of the 1,2-butanediol solution (the $T_{G}$ of the [BEG] is of $220 \mathrm{~K}$ ). It is well-known that in the case of dry powders of nanowires, a $\mathrm{CoO}$ nanometric shell appears on the surface of the Co nanowires giving rise to a characteristic shift of the FC cycles at low temperature. This shift is due to exchange bias effect coming from the coupling of the AFM $\mathrm{CoO}$ or $\mathrm{NiO}$ shell together with the $\mathrm{FM} \mathrm{Co}_{80} \mathrm{Ni}_{20}$ core (author?) 31. Keeping the nanowires in polyol solution avoids any air exposure and therefore prevents metal from oxidation.In this condition we could verify the absence of the exchange bias phenomenon from the magnetization reversal inside the nano-objects (author?) [31] (i.e. no shift of the hysteresis cycles was observed even at low temperature (author?) [33]). A straightforward correlation between the coercive field and the shape and morphology of the nano-objects is thus possible. Below $200 \mathrm{~K}$ the nanowires were (mechanically) blocked in the freezed "mother" solution, allowing to exclude mechanical reversal of the nanowires while applying an external magnetic field. Finally, keeping the nanowires inside the polyol synthesis solution allows to assume non-agglomerated nano-objects and equal spacing (i.e. isotropic interactions) between the nanowires of different batches. This latter characteristic is very useful while comparing static magnetization reversal mechanism among differently-shaped nanowires.

\section{NANOWIRE SHAPE AND LENGTH EFFECTS}

ZFC and FC hysteresis loops at a fixed temperature have been analyzed in terms of coercivity (author?) [33]). Because of the fact that the nanowires were kept in their polyol solution, neither the volume nor the mass of nanowires could be correctly estimated, making it impossible to extract quantitatively the saturation magnetization. Thus, only the coercive field analysis will be reported thereafter. As explained in the previous section, the studied samples presented typical cylindrical to diabolo-like shapes with more or less pronounced head morphology (see Figure 1(a)). We could then study the morphology effect on the magnetization reversal versus nanowire controlled shapes going continuosly from the well defined diabolo (TEM images of the upper line in 


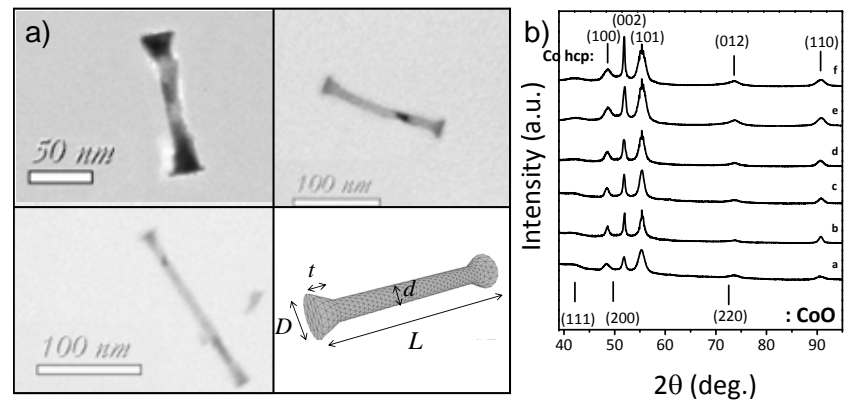

Figure 1: (a) TEM images of various $\mathrm{Co}_{80} \mathrm{Ni}_{20}$ nanowires elaborated under different polyol synthesis conditions; in the right-hand bottom square is reported a schematic mesh reproducing the typical nanowires morphology observed; (b) XRay diffraction patterns for six different batches exhibit the hexagonal symmetry (Co hcp phase is indexed). For these measurements we had to use dry nanopowders and this is why, in some cases, we observed typical diffraction picks of the $\mathrm{CoO}$ phase.

Figure1(a)) to the cylinder type (left-bottom-hand TEM image of Figure1(a)). In a very primitive senario, the coercive field $H_{C}$ in this Co-based one-dimensional systems is mainly driven by an effective anisotropy field composed by two main contributions: i) one belonging to the magnetocrystalline anisotropy $\left(K_{M C}\right)$ and ii) the other related to the shape term $\left(K_{\text {Shape }}\right)$. The first term is expected to be temperature dependent while the second is almost constant as function of temperature (in the range 20-200 K) and only differs for different morphologies (author?) [32. The effective anisotropy acting then on a single nanowire can be written as the sum of the magnetocrystalline and shape contributions if both are aligned with the length of the nanowire. Thus the coercivity can be defined as:

$$
H_{C} \approx H_{M C}(T(K))+H_{\text {Shape }}(L, d, D, t)
$$

In Figure2 (upper graph) typical ZFC and FC coercive behavior is reported for two of the different studied morphologies. The value of the four morphological parameters for the two samples are reported in the graphs. The coercive behavior of both samples versus temperature displays the variation expected for the magnetocrystalline anisotropy contribution. Indeed the coercive field increases with the decreasing temperature. All our nanowires show a pure hcp structure which is usually linked to a high magnetocrystalline term $\left(K_{M C} \approx 5 \times 10^{6}\right.$ erg. $\mathrm{cm}^{-3}$ at room temperature), approximately linearly dependent on temperature. Considering this term equal for all the nanowires, we can then ascribe the observed differences in the coercive field values only to the shape contribution.

Before going through details into this morphology differences, let us comment on another important feature shown in the upper graph of Figure 2, which is the ZFC behavior compared to the FC one. As expected for nonagglomerated nanowires, the FC coercive values are systematically higher than the ZFC ones, proving the efficiency of the nanowires alignment by the magnetic field application during cooling. The two samples proposed in Figure 2 (upper graph) present a substantially different alignment effect under the FC procedure. The gap between the FC and the ZFC coercive values for each sample is appreciable. In the case of longer nanowires (circle symbols) this gap is relatively high (around $1 \mathrm{kOe}$ ) all along the temperature range while in the case of the shorter nanowires (square symbols) the gap is much smaller (around 100Oe). This leads us to conclude that the longer nanowires $(L=150 \mathrm{~nm})$ align better along the magnetic field than the shorter ones. This result is pretty much coherent with a one-dimensional nano-object having the anisotropy axis parallel to the long axis. At this point, we should also notice that the longer nanowires present also smaller diameter $(d=7.6 \mathrm{~nm})$ and smaller head sizes $(D=12 \mathrm{~nm}$ and $t=8.6 \mathrm{~nm})$ which means that their shape is closer to a cylinder, while shorter nanowires are closer to a diabolo shapes. All those characteristics are favorable for the observation of higher coercive field values once the nanowires are aligned along the external applied magnetic field (i.e. FC values)(author?) [2, 4, 22]. These results suggest that when the shape of the nanowires approaches the cylinder type, it is easier to align them along the magnetic field and their coercive values go closer to the expected ones for Co based nanowires (expected value of $8.8 \mathrm{kOe}$ at room temperature). On the contrary, when approaching the diabolo shape (square symbols in Figure 2), the nanowires alignment seems to become less efficient (i.e. the gap between the ZFC and FC curves is smaller) and at the same time coercive field values are few thousands of Oe smaller than the cylinder-type shape values (circle symbols Figure 2).

It is worth noticing at this point that even if we could not extrapolate absolute magnetization values from our measurements for the reason detailed above, we analyzed the $\left(\frac{M_{r}}{M_{S}}\right)$ ratio. We defined as ususal remanence as $M_{r}=M(H=0 k O e)$ and, as the hysteresis cycles have shown fully saturation around $30 \mathrm{kOe}$, we defined the saturation magnetization as $M_{s}=M(H=30 k O e)$ (see inset in Figure2(a) in which only the half-positive part of the hysteresis cycle is shown, the half-negative being symmetrical). We found typical value of $\left(\frac{M_{r}}{M_{S}}\right)$ ratio $\approx 0.5$ for all ZFC hysteresis loops. This latter value is expected for a standard Stoner-Wohlfarth model in the case of random-oriented nanowires (author?) [34]. In the case of FC hysteresis loops, the $\left(\frac{M_{r}}{M_{S}}\right)$ ratio was usually $\geq 0.6$ and reached the value of $\approx 0.7$ for the cylinder type nanowires (circle symbols), confirming the easier alignment for the nanowires with the highest coercivity (see inset 2(a)). We remind here that in the case of nonperfect ellipsoidal morphology the remanence values for magnetically uniform elongated nanoparticles are found 

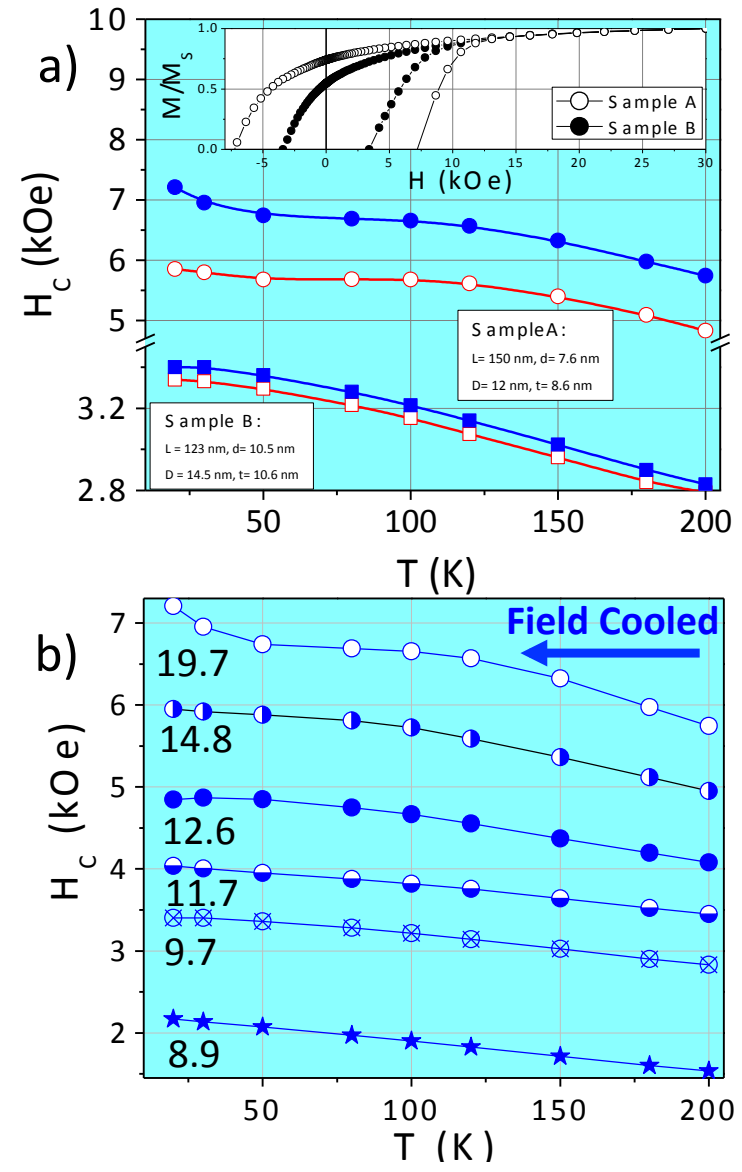

Figure 2: a) ZFC (open symbols) and FC (filled symbols) coercive temperature behavior for two different morphologies (sample A cylinder type (circle symbols) and B diabolo type (square symbols)) among all the studied nanowires (the morphology parameters are reported for each case next to the experimental points); upper inset shows the half-positive hysteresis normalized cycles $\left(\frac{M}{M_{S}}\right)$ for both A and B samples. b) FC coercive behavior for different shaped nanowires (values reported in the left side of the graph represent the $\frac{L}{d}$ ratio for each coercive curve).

to be lower then 1and may be as low as 0.7 in the case of perfectly aligned dumbbells.(author?) [26, 27]. Thus this may explains the small values we observed even for the easier alignment.

For obvious reasons of easy-axis configuration optmized along the applied magnetic field, in order to study the morphology effect on the coercive behavior observed in all our samples, we reported in the bottom graph of the Figure 2 the FC coercive field as a function of temperature for various nanowire shapes. All of them have ZFC coercive fields lower then the FC fields (not shown here), in agreement with the behavior shown in the upper graph and discussed previously. Micromagnetic simulations performed on one-dimensional nano-objects of different shapes showed that the magnetization reversal is strongly dependent of the length over diameter ratio
$\left(\frac{L}{d}\right)$ (author?) [26, 27]. From these previous works, the coercive values in one-dimensional systems are expected to increase with the increasing ratio for a very large range of length and diameter size. This is confirmed by our measurements in the whole range of temperature. This result is strengthened by the fact that the expected behavior is observed for all head-morphology types. This suggests that the ratio $\left(\frac{L}{d}\right)$ is the driving parameter for the coercive field optimization, no matter the head morphology. Thus, so far, the experimental data follow quite well the standard model. In Ref. (author?) [27, micromagnetic simulations have been employed to demonstrate that there is a critical value of the length over diameter ratio above which the coercive field saturates. This result has been validated by simulations of the reversal mechanism of various shapes (i.e. ellipsoid, cylinder, diabolo and dumbbell) and they yielded a critical ratio $\frac{L}{d}=10$. In order to compare our experimental results to this micromagnetic observation, we selected among all the different batches, the samples showing the same head morphology and the same diameter and we inspected their coercive behavior as a function of the length. Figure 3 presents the normalized coercivity as function of the length for diabolo nanowires having the same $d=8$ nm, the same head-width $D=12 \mathrm{~nm}$ and the same headthickness $t=8 \mathrm{~nm}$ (equality are considered within the statistical error of around $\pm 1 \mathrm{~nm}$ ). The coercive field values have been analyzed at low (20K) and high (200K) temperature. These values clearly support the previous micromagnetic simulations (author?) [27] showing that the maximum coercive value is constant for the longest nanowires and start to decrease for $\frac{L}{d} \leq 15$ (10\% loss is observed in Figure 3 at $\frac{L}{d}=15$ ).

\section{HEAD MORPHOLOGY EFFECTS AND CORRECTION FACTOR}

In order to explore the morphology effect on the reversal mechanism in nanomagnets, we decided to analyze the temperature dependence of $H_{C}$ as function of the head morphology ratio $\frac{d}{D}$ (i.e. the width of the conic head base $(D)$ over the diameter taken in the middle of the nanowire $(d))$. We performed this analysis for nanowires with shapes varying from the diabolo-type to the cylinder-type. Figure 4 presents the measured coercive fields as function of $\frac{d}{D}$ ratio. As expected, at a fixed $\frac{d}{D}$ ratio, $H_{C}$ increases as temperature is decreased for each head morphology (see colored symbols in Figure 4). However, an unexpected $H_{C}$-decrease of several $\mathrm{kOe}$ is observed approaching the cylindrical shape while the diabolo-type nanowires (i.e. lower $\frac{d}{D}$ ratio) show higher coercivity values. It is worth mentioning that micromagnetic simulations have already shown that the cylindrical shape is a better geometry than the diabolo one in order to maximize the coercive field (author?) [26]. This is not the case in our experimental observations in which nanowires with more pronounced conic-head (i.e. diabolo 


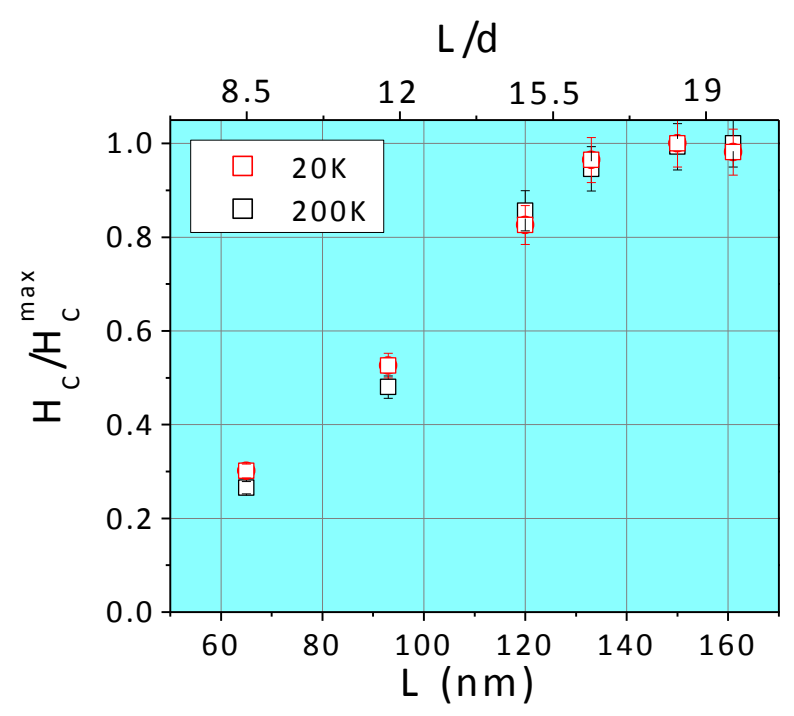

Figure 3: Normalized coercive field at $20 \mathrm{~K}$ (red circles) and $200 \mathrm{~K}$ (black squares) as function of the length for diabolos nanowires having the same head morphology and diameter (i.e. $d=8 \mathrm{~nm}, D=12 \mathrm{~nm}$ and $t=8 \mathrm{~nm}$ ). Note that the values have been normalized to the maximum coercivity observed for the selected batches (i.e. $H_{C}^{\max }=5.8 \mathrm{kOe}$ ). The top scale reports the length over diameter ratio for the different nanowires.

type) present the higher $H_{C}$. At this point, we decided to test the correlation between the $\frac{L}{d}$ ratio and the $\frac{d}{D}$ ratio of the nanowires. $\frac{L}{d}$ is reported as a function of $\frac{d}{D}$ in the inset of Figure 4 . Due to the elaboration parameters explored during the nanowire growth, this two ratios show a tight linked behavior. The shape ratio decreases with the increasing head morphology parameter favoring the growth of a pronounced head in longer nanowires. Looking at the results in the inset of Figure4, we thus expect from the nanowires having the smaller $\frac{d}{D}(\lesssim 0.65)$ ratio to present the higher $H_{C}$ as these latter have also the higher $\frac{L}{d}$ ratio value. Respectively, we expect from the nanowires having the highest $\frac{d}{D}(\lesssim 0.9)$ ratio to present the smaller $H_{C}$ as these latter have also the lower $\frac{L}{d}$ ratio value. In the main figure 4, this is exactly the coercivity behavior observed. From these observations, we can conclude that the head morphology $\left(\frac{d}{D}\right)$ effect on the reversal mechanism, comes directly from the shape $\left(\frac{L}{d}\right)$ effect reported in the previous paragraph. Due to specific growing conditions, the aspect ratio $\frac{L}{d}$ and head morphology parameter $\frac{d}{D}$ are not independent parameters and do not allow us to explore them separately; this could lead to a paradoxical interpretation where a diabolo-shaped nanowire is a better nanomagnet than a cylinder-shaped one.

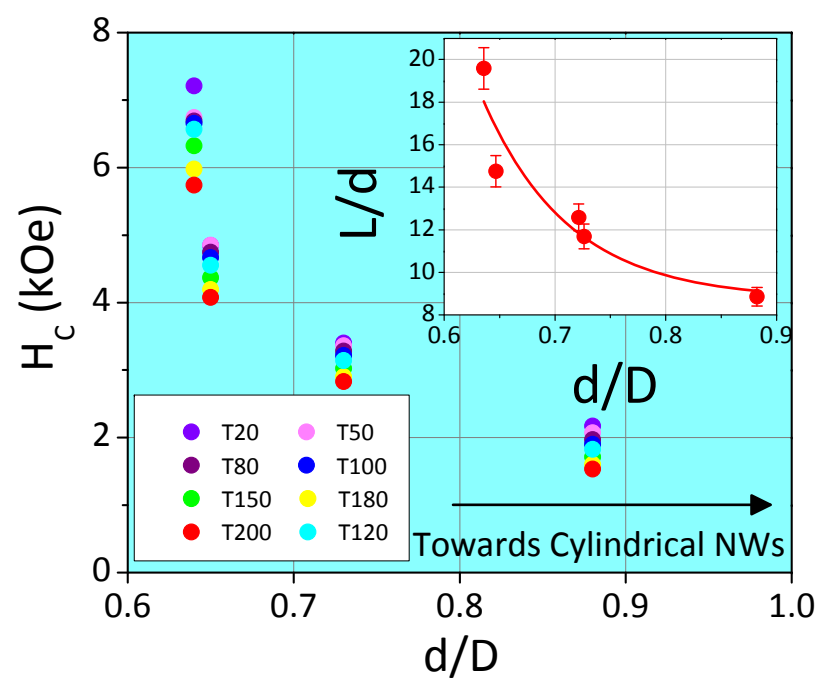

Figure 4: Coercive field $\left(H_{C}\right)$ variations (at different temperature, see symbols for correspondences) as function of the head-morphology ratio $\left(\frac{d}{D}\right)$ showing the paradoxical fact that nanowires with a diabolo shape are better nanomagnets then the cylinder one. The insert corresponds to the one-dimensional shape parameter $\frac{L}{d}$ as function of headmorphology $\frac{d}{D}$ one.

In order to further explore the head morphology effects, we found it opportune to analyze independently the effects of the head thickness $t$. Thus the $H_{C}$-dependence with $t(n m)$ has been studied. Figure 5 a) presents the normalized $H_{C}$ variation as function of $t$. The normalization of $H_{C}$ has been done by using the coercive field of the smaller available thickness $t\left(H_{C}(t=8.6 n m)\right)$ at each temperature. This figure clearly shows that the thicker is the conic head (i.e. higher $t$ ), the higher is the loss in coercivity. Surprisingly enough, after reaching a minimum (around $t=10.5 \mathrm{~nm}$ ) a rise in the coercivity is then observed with increasing $t$.

In order to verify that the driving parameter of the unusual behavior observed in Figure 5 a) is the thickness $t$ of the nanowire head, we performed micromagnetic simulation for nanowires having the same average shape and morphology as the measured ones $(L=150$ $\mathrm{nm}, D=20 \mathrm{~nm}, d=10 \mathrm{~nm})$ and with a thickness $t$ ranging from 0 to $50 \mathrm{~nm}$. The simulations were performed using the NMAG package. Details on procedure and parameters used for these simulations can be found in elsewhere (author?) [27. The magnetic parameters of bulk Co80Ni20 were used during the simulations, namely: magnetization saturation $M_{S}=1.2 * 10^{3} \mathrm{emu} . \mathrm{cm}^{-3}$ and exchange stiffness $A=1.2 * 10^{6} \mathrm{erg.cm}{ }^{-1}$. No magnetocrystalline anisotropy was included to avoid mixing different sources of coercivity. In Figure 5 b), the simulated normalized $H_{C}(t(n m))$ curve qualitatively reproduced the experimental results which proves that the unusual magnetic behavior is driven by the $t$-variation. Previous works(author?) [2, 26, 27] demonstrated that for diabolo 


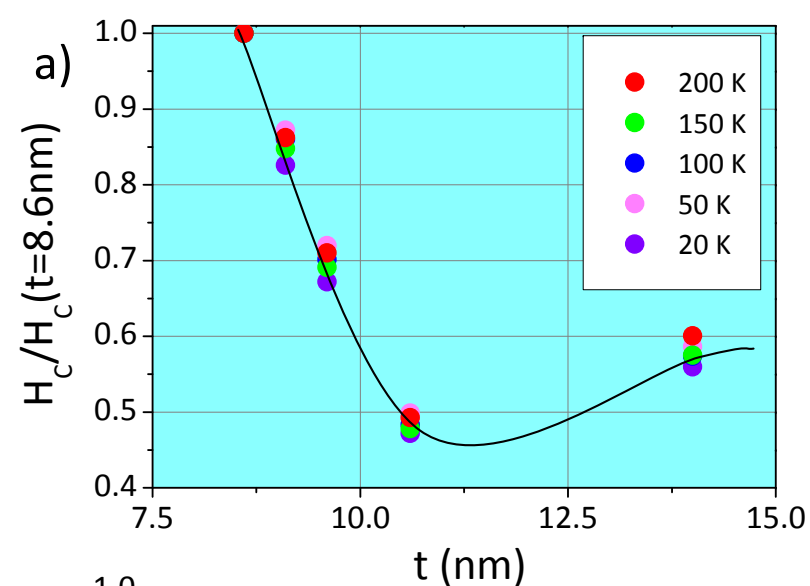

3, 22, 23, 24, 25, 26, 27. These results are, to our knowledge, the first experimental proof of the existence of a morphology factor that corrects the magnetization reversal mechanism mainly driven by the shape aspect ratio. The correction factor $t$ is independent from the aspect ratio $\frac{L}{d}$ and drives the non-trivial behavior observed in Figure 5 .

\section{CONCLUSIONS}

In conclusion we reported the morphology dependence of the magnetization reversal mechanism in weak interacting (non-agglomerated) $\mathrm{Co}_{80} \mathrm{Ni}_{20}$ pure hcp nanowires. Non-agglomerated nanowires with a controlled shape going from a quasi perfect cylinder to a diabolo type have been studied $(D=(8-24) \mathrm{nm}, t=(7-14) \mathrm{nm}$, $d=(7-12) \mathrm{nm})$. The coercive field versus the shape ratio $\left(\frac{L}{d}\right)$ followed a standard expected behavior simulated in previous micromagnetic calculations: $H_{C}\left(\frac{L}{d}\right)$ increases with the increasing ratio. Our results confirm the predominant effect of the shape ratio on the magnetization reversal mechanism inside nanomagnets. Interestingly, an unexpected paradoxical behavior was observed as function of the head morphology $\left(\frac{d}{D}\right) . H_{C}$ decreases with increasing $\frac{d}{D}$, leading to a picture in which a diabolo shaped nanowire is a better nanomagnet than a perfect cylinder. This effect can be interpreted by the strong correlation between the head morphology ratio $\left(\frac{d}{D}\right)$ and the shape aspect one $\left(\frac{L}{d}\right)$. Further experimental analysis and micromagnetic simulations are in excellent agreement showing a peculiar non-monotonous variation of the coercivity field $H_{c}$ with the head thickness parameter $t$. In conclusion, our results show clearly the existence of two independent morphology parameters driving the magnetization reversal mechanism in nanomagnets. This makes it stringent, for nanomagnet application of cobalt based nanowires, to optimize both the dominant shape aspect ratio parameter $\left(\frac{L}{d}\right)$ and the non-trivial head thickness independent one $(t)$.

\section{Acknowledgments}

the head should yield a continuous decrease of the coercivity. Thus the $H_{C}(D(n m))$ theoretical behavior is expected to monotonically decrease without any unexpected minimum. From Figure 5b), we can intuitively understand that the rise of $H_{C}$ at high $t$ is due to the tendency of the nanowires shape to go towards a cylinder having a bigger diameter in the middle $d=D=20 \mathrm{~nm}$ (see schematics in Figure 5 b)). This latter has a lower coercive value (i.e. $\sim 0.6$ times the one of the cylinder having $d=10 \mathrm{~nm}$ ) as reported in previous works(author?) ported through grants from Region Ile-de-France. ANR (AgenceNationale de la Recherche) and CGI (Commissariat Général à l'Investissement) are gratefully acknowledged for their financial support of this work through Labex SEAM (Science Engineering for Advanced Materials and devices) ANR 11 LABX 086, ANR 11 IDEX 05 02 .

[1] S. P. Gubin and Yu. A. Koksharov, Inorganic Materials, 38, 1085 (2002)

[2] L. Sun, Y. Hao, C.-L. Chien and P. C. Searson, IBM Journal of Research and Development, 49, 1 (2005)

[3] J. Zhang, G. A. Jones, T. H. Shen and S. E. Donnely, J. Appl. Phys., 101, 054310 (2007)

[4] K. Soulantica, F. Wetz, J. MaynadiÃ C, A. Falqui, R. P. Tan, T. Blon, B. Chaudret and M. Respaud, App. Phys. 
Lett., 95, 152504 (2009)

[5] Y. Soumare, C. Garcia, T. Maurer, G. Chaboussant, F. Ott, F. Fĩ̃ Cvet, J.-Y. Piquemal and G. Viau, Adv. Funct. Mater., 19, 1971-1977 (2009)

[6] G. Viau, C. Garcia, T. Maurer, G. Chaboussant, F. Ott, Y. Soumare and J.-Y. Piquemal, Phys. Status Solidi A 206, 4, 663 (2009)

[7] J. Sanchez-Barriga, M. Lucas, F. Radu, E. Martin, M. Multigner, P. Marin, A. Hernando and G. Rivero, Phys. Rev. B 80, 184424 (2009)

[8] L.G. Vivas, M. Vazquez, J. Escrig, S. Allende, D. Altbir, D. C. Leitao and J.P. Araujo, Phys. Rev. B 85, 035439 (2012)

[9] W. Fang, I. Panagiotopoulos, F. Ott, F. Bou $\widetilde{C}$, K. Ait-Atmane, J.-Y. Piquemal, G. Viau and F. Dalmas, J. Nanopart. Res. 16, 2265 (2014)

[10] A. Akbarzadeh, M. Samiei and S. Davaran, Nanoscale Research Letters 7, 144, (2012)

[11] D. Weller and A. Moser, IEEE Trans. Magn., 35, 4423 (1999)

[12] K. Gandha, K. Elkins, N. Poudyal, X. Liu and J. Ping Liu, Scientific Reports 4, 5345 (2014)

[13] N. Ouar, F. Schoenstein, S. Mercone, S. Farhat, B. Villeroy, B. Leridon and N. Jouini, J. Appl. Phys. 114, 163907 (2013)

[14] J. Meier, B. Doudin and J.-Ph. Ansermet, J. Appl. Phys. 79, 6010 (1996)

[15] G. C. Han, B. Y. Zong, P. Luo, and Y. H. Wu, J. Appl. Phys. 93, 9202 (2003)

[16] D. J. Sellmyer, M. Zheng and R. Skomski, J. Phys.: Cond. Matter 13, R433 (2001)

[17] M. Vazquez, M. Hernandez-Velez, K. Pirota, A. Asenjo, D. Navas, J. Velazquez, P. Vargas and C. Ramos, Eur. Phys. J. B 40, 489 (2004)

[18] S. J. Hurst, E. K. Payne, L. Qin and C. A. Mirkin, Angew. Chem. Int. Ed. 45, 2672 (2006).

[19] Q. Liu, X. Guo, J. Chen, J. Li, W. Song and W. Shen, Nanotechnology 19, 365608 (2008).

[20] F. Dumestre, B. Chaudret, C. Amiens, M.-C. Fromen, M.
J. Casanove, P. Renaud and P. Zurcher, Angew. Chem. Int. Ed. 41, 22 (2002)

[21] F. Dumestre, B. Chaudret, C. Amiens, M. Respaud, P. Fejes, P. Renaud and P. Zurcher, Angew. Chem. Int. Ed. 42, 5213 (2003)

[22] H. Zeng, M. Zheng, R. Skomski, D. J. Sellmeyer, Y. Liu, L. Menon and S. Bandyopadhyay, J. Appl. Phys. 87, 4718-4720 (2000)

[23] R.P. Cowburn, J. Phys. D: Appl. Phys. 33, R1 (2000)

[24] D. Ung, Y. Soumare, N. Chakroune, G. Viau, M.-J. Vaulay, V. Richard and F. Fiévet, Chem. Mater. 19, 2084 (2007)

[25] A Gaul, N Ouar, S Mercone, F Zighem, F Schoenstein, B Leridon, S Farhat, N Jouini, MRS Bulletin 01/2014; 1708:mrss14-1708-vv06-08

[26] F. Ott, T. Maurer, G. Chaboussant, Y. Soumare, J.-Y. Piquemal, G. Viau, J. of Appl. Phys. 105, 013915 (2009)

[27] F. Zighem and S. Mercone, J. of Appl. Phys 116, 193904 (2014)

[28] V. Sokalski, D. E. Laughlin and J.-G. Zhu, J. Appl. Phys. 110, 093919 (2011)

[29] C.J. Aas, L. Szunyogh, R.F.L. Evans and R.W. Chantrell,, J. Phys.: Cond. Matter 25, 296006 (2013)

[30] F. Fiévet, J.P.Lagier, B. Blin, B. Beaudoin, M. Figlarz, Solid State Ionics, 32, 198(1989)

[31] T. Maurer, F. Zighem, F. Ott, G. Chaboussant, G. André, Y. Soumare, J.- Y. Piquemal, G. Viau, C. Gatel, Physical Review B, 80, 064427 (2009)

[32] K. Ait Atmane, F. Zighem, Y. Soumare, M. Ibrahim, R. Boubekri, T. Maurer, J. Margueritat, J.-Y. Piquemal, F. Ott b, G. Chaboussant, F. Schoenstein, N. Jouini, G. Viau, J. Sol. St. Chem. 197, 297 (2013)

[33] See supplemental material at [URL will be inserted by AIP] for magnetic hysteresis cycles and coercive fields table at all temperatures and for nanowires shape and head morphology statistics.

[34] T. Maurer, F. Ott,G. Chaboussant, Y. Soumare, J.-Y. Piquemal, G. Viau, Appl. Phys. Lett. 91, 172501 (2007) 